\title{
Subluminal Cosmology
}

\author{
Rainer Burghardt \\ Independent Researcher, Obritz, Austria \\ Email:arg@aon.at
}

How to cite this paper: Burghardt, R. (2017) Subluminal Cosmology. Journal of Modern Physics, 8, 583-601.

https://doi.org/10.4236/jmp.2017.84039

Received: February 6, 2017

Accepted: March 25, 2017

Published: March 28, 2017

Copyright $\odot 2017$ by author and Scientific Research Publishing Inc. This work is licensed under the Creative Commons Attribution International License (CC BY 4.0).

http://creativecommons.org/licenses/by/4.0/

\begin{abstract}
The standard model of cosmology is considered critically. A model with pressure is proposed which is linearly expanding and which is an exact solution of Einstein's field equations. The recession velocity of the galaxies of this model never exceeds the speed of light. The model is closely related to the $R_{h}=c t$ model of Melia, which is flat and infinite. However, our subluminal model is spatially positively curved and closed. Nevertheless all data from observations gathered and surveyed by Melia support our model.
\end{abstract}

\section{Keywords}

Expanding Cosmos with Pressure, Subluminal Expansion, Covariant Representation

\section{Introduction}

The standard model (StM) is based on the pressure-free Friedman model which has been extended with relations involving pressure. Although this model is largely based on astrophysical considerations, it must be mentioned that it has some deficiencies that limit the usability of the model. First, we will list these deficiencies and then we will investigate whether some of these deficiencies can be avoided by improving the model or by applying new strategies.

1) Pressure is inserted by hand into the Friedman model. The StM is not an exact solution to Einstein's field equations.

2) Since one cannot determine all the variables with Einstein's field equations, five parameters have to be adapted in such a way that a good agreement with data collected by observation is possible.

3) The method described above shows that our universe is almost flat, but that it is nevertheless homogeneous and closed and therefore must contain a very large number of galaxies.

4) The theory needs a deceleration parameter that contains second derivatives of the scale factor with respect to time. 
5) This means that accelerations are possible. The time-like metric coefficient of the metric of the StM given in comoving coordinates is $g_{44}=1$. This means that the cosmos expands in free fall. In a free-falling system no accelerations can be observed according to the theory of general relativity. Thus, the StM violates the principles of general relativity.

6) By a motion with superluminal velocity, galactic island formation can occur. Galaxies are causally separated from each other. No information exchange can take place between them.

7) The Hubble law suggests a Galilean velocity addition.

8) In order to reconcile the StM with the data, it must be assumed that soon after the Big Bang inflation, i.e. a temporary expansion with approximately 500 times the light speed must have taken place.

In Section 2, we will consider some of the topics mentioned. In Section 3, we examine the problem of Einstein's elevator in the context of free-falling observers in the expanding cosmos. In Section 4 we describe a subluminal model based on a previous paper [1], a model which is an exact solution to Einstein's field equations, which contains pressure, and which does not allow superluminal velocities. In Section 5 we treat the field equations of the model in the comoving system, in Section 6 in the non-comoving system. In Section 7 we discuss the special properties of the model, the recession velocities, the cosmic horizon and the relation to the model of Melia.

\section{Preliminary Remarks}

We believe that Nature is not so cruel as to describe the world by an incomplete system of equations. In [1] we have proposed a model which is based on an exact solution of Einstein's field equations and which in addition describes the pressure of the cosmic fluid. First we have presented our subluminal model only as a possibility of thinking. The model of Melia [2]-[20] is actually flat, but in some cases it is consistent with our spatially positively curved model. Thus, we are encouraged to present our proposal as a realistic model. Melia and his co-workers have shown that the data observed fit better into their model than the StM or other FRW models and without any adjustment. If one reinterprets Melia's model $(\mathrm{MeM})$, the identity with our subluminal model is ensured. All the statements made by Melia about observed data and their fitting apply equally to the subluminal model (SuM).

Although it is emphasized according to previous observations that one cannot say whether the universe is positively curved, flat, or negatively curved, we assume that the SuM is spatially positively curved. In addition, we do not propose that it is almost flat, as is deduced from the StM.

Melia calls his model $R_{h}=c t$-model. It has been questioned by some authors [21]-[27], chiefly concerning the cosmic horizon. In one of his papers Melia [16] has presented convincing arguments against these objections.

With the subluminal model, we also avoid the conception that, as is the case with the version of Melia, the flat nevertheless homogeneous universe contains 
infinitely many stars. We do not need to answer the question as to how and when these infinite stars have been created. A closed universe can only contain a finite number of stars.

The MeM as well as the SuM does not contain a deceleration parameter, thus no second derivatives of the scale factor with respect to the time. The expansion of the cosmos is uniform, no acceleration occurs. Most cosmological models assume that expansion comes about in free fall [28]. This is reflected in the form of the metric. The timelike metric coefficient in comoving coordinates is $g_{44}=1$ or at least position-independent. From it no accelerations can be derived. The fact that a deceleration parameter is still contained in such models follows from the supplement of the Friedman model by pressure. This extension takes place by hand and thus the model is not an exact solution to Einstein's field equations.

One of the most remarkable features of the StM is the fact that it allows superluminal velocities. The recession velocity of the galaxies reaches the speed of light at the Hubble horizon, at this location the red shift of the light is infinitely high, and signal transmission is no longer possible. Beyond this horizon the galaxies move faster than light. This causes galaxies and galaxy clusters to separate. They do not have any connection to each other and a galactic island formation will take place. Thus, the StM allows to violate the basic principles of the special theory of relativity. This interpretation is favored by Davis [29] and Davis and Lineweaver [30]. In these considerations it is important whether everything is expanding in the universe or whether local areas such as our solar system or atomic areas are excluded. With this problem the following researchers have been concerned: Davis and Lineweaver [31], Anderson [32], Blau [33], Callender and Weingard [34], Carrera [35], Coooperstock, Faraoni, and Vollick [36], Dicke and Peebles [37], Irvine [38], Mizony and Lachièze-Rey [39], and Sereno and Jetzer [40]. Remarks on the recession velocity can be found in Chodorowski [41], Cook [42], Endean [43], Harrison [44], Kiang [45], Lewis et al. [46], Murdoch [47], Silverman [48], Stuckey [49] and Liebscher [50]. On the horizons the following researchers have made contributions: Rindler [51], Barnes, Francis, James, and Lewis [52], Ellis and Rothman [53], Ellis and Stoeger [54], Harrison [55], Shi and Turner [56]. Some authors claim that the redshift is not due to the extension of the space, but to the relative motion of the stars, i.e. to the associated Doppler effect: Whiting [57], Harvey, Schucking, and Surowitz [58], Bunn and Hogg [59], Chodorowski [60] [61], Faraoni [62] and Narlikar [63]. The gravitational effect of the stars could also influence the wavelength of the light: Bondi [64], Endean [65], Infeld and Schild [66] and Querella [67]. Thus, it is not necessary to introduce the expansion of the cosmos in order to explain the redshift: In a static cosmos, the stars move away from each other. Abramowicz, Bajtlik, Lasota, and Moudens [68] [69] have decisively opposed this point of view. They argue that it can be decided by observation whether the cosmos expands or the redshift can be explained by kinematic effects. Nevertheless, some authors are inclined to this view: Aspden [70], Chodorowski [71] [72], Epstein [73], Felten and Isaacman [74], Harvey [75], Peacock [76], and Stuckey [77]. 
Olbers' paradox gets little attention in the literature. In an infinite universe with an infinite number of stars an infinite amount of light is emitted. But in this case only a finite amount arrives at us, still so much that the night sky would be as bright as our sun. Expanding cosmological models which have a horizon prevent the radiation of light from this region and thus solve Olbers' paradox: Harrison [78], Wesson, Valle, and Strabell [79] and Wesson [80].

These often very detailed discussions obviously arise from the discomfort which causes the StM among astrophysicists. In the next Section, we will examine the influence on the form of the metric of a cosmos which expands in free fall. For the purpose of understanding, it is also necessary to recognize the relation between the curvature parameter $\mathrm{k}$ and the curvature of the space.

\section{Expansion in Free Fall}

Most expanding models, among them the StM, expand in free fall. The metrics of the models are generally written in comoving coordinates. The metric in this representation is quite simple and can easily be further processed

$$
\mathrm{d} s^{2}=K^{2}\left[\frac{1}{1-k \frac{r^{\prime 2}}{R_{0}^{2}}} \mathrm{~d} r^{\prime 2}+r^{\prime 2} \mathrm{~d} \Omega^{2}\right]-\mathrm{d} t^{\prime 2} .
$$

Therein $K$ is the position-independent but time-dependent scale factor, $\Omega$ the solid angle, and $k$ the curvature parameter, which can take the values 1,0 , -1 according to the FRW classification. For $k=1$ the spatial curvature of the cosmos is positive and the cosmos is closed. A space with $k=0$ is called a flat open space and with $k=-1$ a negatively curved and open cosmos. $R_{0}$ is a constant that can be absorbed by $r^{\prime} .\left\{r^{\prime}, t^{\prime}\right\}$ are comoving coordinates, in particular $t^{\prime}$ is the cosmic time which applies equally to all observers. Since the metric factor is $g_{4^{\prime} 4^{\prime}}=1$, the coordinate time coincides with the proper time of comoving observers. The representation in comoving coordinates has the advantage that the field equation system substantially simplifies. The form (3.1) of the metric is called a canonical form.

We are critical concerning the interpretation of the quantity $k$ as the curvature parameter, and have communicated this in some papers [1] [81] [82]. Above all, we want to doubt that $k=0$ stands necessarily for a flat space. One of the fundamentals of the general theory of relativity is that a gravitational attraction cannot be experienced in a freely falling system. Observers in a freely falling elevator hover. Since there seem to be no gravitational forces, they could opine the space to be flat, thus, that $k=0$ is valid. Therefore, they could assess the space to be flat, although the curvature of the space did not change due to the falling motion. Exactly this reasoning has been lost to cosmology. In a cosmos that expands in free fall, no gravitational forces are experienced. If its metric in the comoving coordinate system reads as

$$
\text { (A) } \mathrm{d} s^{2}=\mathrm{K}^{2}\left[\mathrm{~d} r^{\prime 2}+r^{\prime 2} \mathrm{~d} \Omega^{2}\right]-\mathrm{d} t^{\prime 2} \text {, }
$$


it is of the type $k=0$ and the cosmos is assumed to be flat and spatially infinite. The fact that a metric of type (A) can describe a cosmos that is positively curved and spatially closed is shown by the de Sitter cosmos and by our subluminal model. The metric of the static dS cosmos has the canonical form

$$
\text { (B) } \quad \mathrm{d} s^{2}=\frac{1}{1-\frac{r^{2}}{R_{0}^{2}}} \mathrm{~d} r^{2}+r^{2} \mathrm{~d} \Omega^{2}-\left(1-\frac{r^{2}}{R_{0}^{2}}\right) \mathrm{d} t^{2}
$$

and thus is of type $k=1$. The cosmos is spatially positively curved. The coordinate system $\{r, t\}$ is the static one. The positive force $U_{m}=\left\{U_{1}, 0,0,0\right\}$ is derived from the metric coefficient $g_{44}$. It acts at any point of the universe and tries to push away neighboring points. With the transformation of Lemaitre, the metric (B) can be transformed into the form (A), whereby the structure of the space is not changed. To this coordinate transformation a Lorentz transformation can be assigned which leads to a reference system which gives way to the forces $U$. The new system is expanding. Mathematically, this is reflected in the relation

$$
\text { ' } U_{m^{\prime}}=L_{m^{\prime}}^{m} U_{m}+L_{m^{\prime}} .
$$

We call it Einstein's elevator equation. $L_{m^{\prime}}^{m}$ is the matrix of a Lorentz transformation, ' $L_{m}$ ' the Lorentz term. It arises from the inhomogeneous transformation law of the Ricci-rotation coefficients which determine the geometry. Details of this problem have been dealt with in the papers [1] [81] [82]. Particularly reasonable is the first component of Equation (3.4)

$$
{ }^{\prime} U_{1^{\prime}}=U_{1^{\prime}}+{ }^{\prime} L_{1^{\prime}}=0 \text {. }
$$

The driving force $U$ of the static system is canceled by the dynamic term ' $L$. The system expanding in free fall in the $\mathrm{dS}$ cosmos is free of forces. Although the quantity ' $U$ does not have a radial component, it gets a time-like part by (3.4). All in all one has

$$
\left\{U_{1}, 0,0,0\right\} \rightarrow\left\{0,0,0,{ }^{\prime} U_{4^{\prime}}\right\} .
$$

Thus, the same considerations as for the free fall in the Schwarzschild field apply to the dS cosmos. By no means a metric of type (A) needs not to describe a flat space. It stands for a reference system which is in free fall.

Similar mechanisms apply to other cosmological models, also for our subluminal model. In the latter case, the situation is somewhat more complicated and it will be dealt with in more detail in the next Section.

\section{The Subluminal Model}

The model [1] which we have proposed earlier, we will here present in a lucid form and we also will elaborate further details. We start from the static dS model which is based on a pseudo-hyper sphere, embedded in a flat 5-dimensional space. We generalize the model by dropping the condition that the radius of the pseudo-hyper sphere is constant and we put

$$
R=R(t)
$$


This leads to a genuine expanding cosmos, whose stress-energy-momentum tensor contains pressure and mass density. At any time of the expansion the geometry of our model is the geometry of the $\mathrm{dS}$ model. Therefore, we will not make straightaway use of the definition (4.1), but we will first explain the fundamentals of the $\mathrm{dS}$ cosmos.

The above-mentioned pseudo-hyper sphere has the embedding in a flat 5dimensional space with the Cartesian coordinates $x^{a}, a=0,1, \cdots, 4$

$$
\begin{aligned}
& x^{3}=\mathrm{R} \sin \eta \sin \vartheta \sin \varphi \\
& x^{2}=\mathrm{R} \sin \eta \sin \vartheta \cos \varphi \\
& x^{1}=\mathrm{R} \sin \eta \cos \vartheta \\
& x^{4}=\mathrm{R} \cos \eta \sin i \psi \\
& x^{0}=\mathrm{R} \cos \eta \cos i \psi
\end{aligned}
$$

wherein $\{R, \eta, \vartheta, \varphi, i \psi\}$ are the quasi-spherical coordinates. On the surface of the pseudo-hyper sphere the metric in these coordinates is given by

$$
\text { (B) } \quad \mathrm{d} s^{2}=R^{2} \mathrm{~d} \eta^{2}+R^{2} \sin ^{2} \eta \mathrm{d} \vartheta^{2}+R^{2} \sin ^{2} \eta \sin ^{2} \vartheta \mathrm{d} \varphi^{2}+R^{2} \cos ^{2} \eta \mathrm{d} i \psi^{2}
$$

With the radial coordinate and the coordinate time

$$
r=R \sin \eta, \quad \mathrm{d} x^{4}=i \mathrm{~d} t=R \mathrm{~d} i \psi
$$

and $\operatorname{Rd} \eta=\mathrm{d} r / \cos \eta$ first

$$
\left(B^{\prime}\right) \quad \mathrm{d} s^{2}=\frac{1}{\cos ^{2} \eta} \mathrm{d} r^{2}+r^{2} \mathrm{~d} \vartheta^{2}+r^{2} \sin ^{2} \vartheta \mathrm{d} \varphi^{2}-\cos ^{2} \eta \mathrm{d} t^{2}
$$

is derived from it. Finally, one obtains the metric in canonical form

$$
\mathrm{d} s^{2}=\frac{1}{1-r^{2} / R^{2}} \mathrm{~d} r^{2}+r^{2} \mathrm{~d} \vartheta^{2}+r^{2} \sin ^{2} \vartheta \mathrm{d} \varphi^{2}-\left(1-r^{2} / R^{2}\right) \mathrm{d} t^{2} .
$$

Performing the Lemaitre transformation the metric in the comoving system obtains the form (3.2). However, considering (4.1), the scale factor of the subluminal model has a form different from that of the expanding $\mathrm{dS}$ model. We will elaborate this expression step by step.

First we will work with the metric (3.2) in the simpler comoving system and we will return later to the non-comoving system. From the metric (A) we read the 4-bein system

$$
\stackrel{1^{\prime}}{e_{1}^{\prime}=K, \quad e^{\prime}} e_{2^{\prime}}^{\prime}=K r^{\prime}, \quad{\stackrel{3}{e^{\prime}}}_{3^{\prime}}^{\prime}=K r^{\prime} \sin \vartheta, \quad \stackrel{4}{e}_{4^{\prime}}^{\prime}=1
$$

Furthermore, the non-comoving radial coordinate $r$ is connected with the comoving $r^{\prime}$ by

$$
r=K r^{\prime} .
$$

From (4.7) we calculate the Ricci-rotation coefficients using the general formula

$$
A_{m n}{ }^{s}=\stackrel{s}{e}_{i} \underset{[n \mid m]}{e^{i}}+g^{s r} g_{m t} e_{i}^{t} \underset{[n \mid r]}{e^{i}}+g^{s r} g_{n t} e_{i[m \mid r]}^{t} \underset{e^{i}}{e^{i}}
$$


however, for the primed system (4.7). We split ${ }^{1}$ the Ricci-rotation coefficients into the following components

$$
\text { ' } A_{m^{\prime} n^{\prime}}{ }^{s^{\prime}}=B_{m^{\prime} n^{\prime}}{ }^{s^{\prime}}+C_{m^{\prime} n^{\prime}}{ }^{s^{\prime}}+U_{m^{\prime} n^{\prime}}{ }^{s^{\prime}} .
$$

With the orthogonal unit vectors of the comoving system

$$
\text { ' } m_{m^{\prime}}=\{1,0,0,0\}, \quad b_{m^{\prime}}=\{0,1,0,0\}, \quad c_{m^{\prime}}=\{0,0,1,0\}, \quad ' u_{m^{\prime}}=\{0,0,0,1\}
$$

we can further disassemble

$$
\begin{gathered}
B_{m^{\prime} n^{\prime}}{ }^{s^{\prime}}=b_{m^{\prime}} B_{n^{\prime}} b^{s^{\prime}}-b_{m^{\prime}}, b_{n^{\prime}} B^{s^{\prime}}, \quad C_{m^{\prime} n^{\prime}}{ }^{s^{\prime}}=c_{m^{\prime}} C_{n^{\prime}} c^{s^{\prime}}-c_{m^{\prime}} c_{n^{\prime}} C^{s^{\prime}}, \\
U_{m^{\prime} n^{\prime}}^{s^{\prime}}={ }^{\prime} m_{m^{\prime}}{ }^{\prime} U_{n^{\prime}} m^{s^{\prime}}-{ }^{\prime} m_{m^{\prime}}{ }^{\prime} m_{n^{\prime}}{ }^{\prime} U^{s^{\prime}} .
\end{gathered}
$$

$B$ and $C$ are the lateral field quantities of the model and ' $U$ is a time like quantity still to be discussed.

A look at the metric (A) and the 4-beine (4.7) shows that we are essentially concerned with a spherically symmetric problem, the treatment of which does not pose a particular problem concerning the field equations. Only the scale factor is a time-dependent variable which describes the change of the pseudo-hyper sphere and cannot be determined from the properties of the pseudo-hyper sphere. For the time-like parts of the Ricci-rotation coefficients one obtains after a short calculation

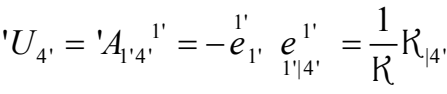

$$
\begin{aligned}
& B_{4^{\prime}}=A_{2^{\prime} 4^{\prime}}^{2^{\prime}}=-\stackrel{2}{e}_{2^{\prime}}^{2^{\prime}} \underset{2^{\prime} \mid 4^{\prime}}{2^{2^{\prime}}}=\frac{1}{K r^{\prime}}\left(K r^{\prime}\right)_{\mid 4^{\prime}}=\frac{1}{K} K_{\mid 4^{\prime}}
\end{aligned}
$$

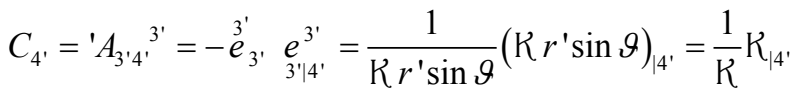

To approach the problem, let us assume that the radius of the pseudo-hyper sphere as well as the radial coordinate in (4.8) are subject to expansion and so we write

$$
R=K R_{0},
$$

where $R_{0}$ is a constant. Furthermore, we can see that in the calculation of the Ricci-rotation coefficients there occurs the time derivative of the scale factor and therefore we define a quantity

$$
F_{m^{\prime}}=\frac{1}{R} R_{\mid m^{\prime}}=\frac{1}{K} K_{\mid m^{\prime}}=\left\{0,0,0, \frac{1}{K} K_{\mid 4^{\prime}}\right\} .
$$

This shows that the expansion scalar

$$
\text { ' } u_{\| s^{\prime}}^{s^{\prime}}={ }^{\prime} A_{r^{\prime} s^{\prime}} r^{\prime} \cdot u^{s^{\prime}}={ }^{\prime} A_{r^{\prime} 4}{ }^{r^{\prime}}={ }^{\prime} A_{4}
$$

is composed of three equal contributions

$$
\text { ' } U_{4^{\prime}} \stackrel{*}{=} B_{4^{\prime}} \stackrel{*}{=} C_{4^{\prime}}=\frac{1}{\mathrm{~K}} \mathrm{~K}_{14^{\prime}},
$$

and that the expansion of the cosmos in the three spatial directions is equal.

${ }^{1}$ Details for the calculation with the tetrad method can be found in papers published about 1900 by Ricci, Bianchi, Levi-Civita, furthermore by Treder [83], Liebscher and Treder [84], and also in our paper [85]. 
With

$$
\partial_{1^{\prime}}=\frac{1}{K} \frac{\partial}{\partial r^{\prime}}, \quad \partial_{2^{\prime}}=\frac{1}{K r^{\prime}} \frac{\partial}{\partial \vartheta}=\frac{1}{r} \frac{\partial}{\partial \vartheta}
$$

the following relations

$$
B_{\alpha^{\prime}}=\left\{\frac{1}{r}, 0,0\right\}, \quad C_{\alpha^{\prime}}=\left\{\frac{1}{r}, \frac{1}{r} \cot \vartheta, 0\right\}, \quad \alpha^{\prime}=1^{\prime}, 2^{\prime}, 3^{\prime}
$$

result for the spatial components of the Ricci-rotation coefficients, relations which are known from a flat geometry with a polar reference system. As already indicated in Section 3, this does not mean that the space is flat, but that the reference system is in free fall. We will discuss this more closely later.

\section{The Field Equations in the Comoving System}

We use the Ricci rotation coefficients to calculate the field equations in the tetrad calculus. Most of the cosmological models are based on spherical symmetric spaces, or at least on spaces with a symmetry that is parameterized with spherical coordinates. Since the mathematical treatment of these spaces is simple, the cosmologists mostly concentrate only on the Friedman equation because it makes the essential statements about the temporal change of the universe. However, we want to work through the field equations system completely, because the spherical-symmetric part of the field equations also provides information, in particular in regard to the curvature of the space.

By means of the relations (4.10) and (4.12) the Ricci

$$
\begin{aligned}
& R_{m^{\prime} n^{\prime}}={ }^{\prime} A_{m^{\prime} n^{\prime} \mid s^{\prime}}^{s^{\prime}}-{ }^{\prime} A_{n^{\prime} \mid m^{\prime}}-{ }^{\prime} A_{r^{\prime} m^{\prime}}^{s^{\prime}} \cdot A_{s^{\prime} n^{\prime}}^{r^{\prime}}+{ }^{\prime} A_{m^{\prime} n^{\prime}}{ }^{s^{\prime}} A_{s^{\prime}}, \quad \text { ' } A_{n^{\prime}}={ }^{\prime} A_{r^{\prime} n^{\prime}}{ }^{r^{\prime}} \\
& R_{m^{\prime} n^{\prime}}=-\left[U^{\prime} U^{s^{\prime}}{ }_{1}+{ }^{\prime} U^{s^{\prime}} \cdot U_{s^{\prime}}\right] h_{m^{\prime} n^{\prime}} \\
& -\left[B_{n^{\prime} \| m^{\prime}}+B_{n^{\prime}} B_{m^{\prime}}\right]-b_{n^{\prime}} b_{m^{\prime}}\left[B_{2}^{s^{\prime}}{ }_{2}^{s^{\prime}}+B^{s^{\prime}} B_{s^{\prime}}\right] \\
& -\left[C_{\substack{n^{\prime} \| m^{\prime} \\
3}}+C_{n^{\prime}} C_{m^{\prime}}\right]-c_{n^{\prime}} c_{m^{\prime}}\left[C_{\substack{\| s^{\prime} \\
s^{\prime}}}+C^{s^{\prime}} C_{s^{\prime}}\right]
\end{aligned}
$$

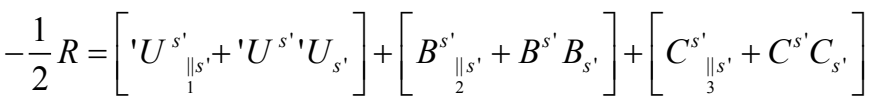

can be decomposed into subequations for the three variables ' $U, B$, and $C$. Therein $h_{m^{\prime} n^{\prime}}=\operatorname{diag}(1,0,0,1)$ is a sub-matrix of the tetrad metric $g_{m^{\prime} n^{\prime}}=\operatorname{diag}(1,1,1,1)$. We are using the graded derivatives as in an earlier paper [82]

$$
\begin{gathered}
U_{n^{\prime} \| m^{\prime}}={ }^{1} U_{n^{\prime} \mid m^{\prime}}, \quad B_{n^{\prime} \| m^{\prime}}=B_{n^{\prime} \mid m^{\prime}}-{ }^{\prime} U_{m^{\prime} n^{\prime}}{ }^{s^{\prime}} B_{s^{\prime}} \\
C_{\substack{n^{\prime} \| m^{\prime} \\
3}}=C_{n^{\prime} \mid m^{\prime}}-{ }^{\prime} U_{m^{\prime} n^{\prime}}{ }^{s^{\prime}} C_{s^{\prime}}-B_{m^{\prime} n^{\prime}}{ }^{s^{\prime}} C_{s^{\prime}}
\end{gathered}
$$

with which the field equations can be clearly exposed. The field equations are solved with the ansatz 


$$
\frac{1}{\mathrm{R}} \mathrm{R}_{14^{\prime}}=-\frac{i}{\mathrm{R}}, \quad \widetilde{F}_{4^{\prime}}=-\frac{i}{\mathrm{R}}
$$

which, as we shall see later on, is confirmed by the conservation law. That means that all field quantities of the system are known

$$
{ }^{\prime} U_{m^{\prime}}=\left\{0,0,0,-\frac{i}{\mathrm{R}}\right\}, \quad B_{m^{\prime}}=\left\{\frac{1}{r}, 0,0,-\frac{i}{\mathrm{R}}\right\}, \quad C_{m^{\prime}}=\left\{\frac{1}{r}, \frac{1}{r} \cot \vartheta, 0,-\frac{i}{\mathrm{R}}\right\}
$$

The quantity ' $U$ does not contain a radial component. According to (4.7) one has

$$
{ }^{\prime} U_{1^{\prime}}={ }^{\prime} A_{4^{\prime} 1^{\prime}}{ }^{\prime \prime}=-\stackrel{4}{e}_{4^{\prime}} e_{4^{\prime} 1^{\prime}}^{4^{\prime}}=0
$$

This means that the universe is in free fall and that no radial forces occur.

With the help of (5.2) the subequations of Einstein's field Equation (5.1) can be calculated

$$
\begin{aligned}
& U_{\substack{{ }_{1}^{\prime} \\
s^{\prime}}}+U^{s^{\prime}} \cdot U_{s^{\prime}}=0 \\
& B_{m^{\prime} \| n^{\prime}}+B_{m^{\prime}} B_{n^{\prime}}=-m_{m^{\prime}}{ }^{\prime} m_{n^{\prime}} \frac{1}{\mathrm{R}^{2}}, \quad B_{\substack{s^{\prime} \\
s^{\prime}}}+B^{s^{\prime}} B_{s^{\prime}}=-\frac{1}{\mathrm{R}^{2}} \\
& C_{m^{\prime} \| n^{\prime}}+C_{m^{\prime}} C_{n^{\prime}}=-\left({ }^{\prime} m_{m^{\prime}}{ }^{\prime} m_{n^{\prime}}+b_{m^{\prime}}, b_{n^{\prime}}\right) \frac{1}{\mathrm{R}^{2}}, \quad C_{\substack{\| s^{\prime} \\
s^{\prime}}}+C^{s^{\prime}} C_{s^{\prime}}=-\frac{2}{\mathrm{R}^{2}}
\end{aligned}
$$

The first relation in it is the Friedman equation. With $\partial_{4^{\prime}}=\partial / i \partial T^{\prime}$, where $T^{\prime}$ corresponds to the proper time of the comoving observer, it can be converted into the form familiar to cosmologists

$$
\frac{1}{R} R^{\cdot}-\frac{1}{R}=0, \quad R^{*}=1, \quad R^{*}=0 .
$$

The rate of expansion of the subluminal model is constant. Recent measurements of the redshift of supernovae indicate an acceleration of the expansion of the cosmos. However, the data have been adapted to the less convincing FRW model. The discussion on this topic, however, is by no means completed.

As stated at the outset, conceptual difficulties arise, if an accelerated expansion is to be considered on the basis of a model which expands in free fall. In the comoving system one has $g_{4^{\prime} 4^{\prime}}=1$. No accelerations can be derived from such a metric. The principle of Einstein's elevator applies. This principle is called "Weak Einstein equivalence Principle" (WEEP) in modern literature [86] [87] [88]. We have illuminated the problem on the basis of the de Sitter cosmos in Section 3. For a model with accelerated expansion a metric with $g_{4^{\prime} 4^{\prime}}=g_{4^{\prime} 4^{\prime}}(r)$ would be expected. Such an ansatz is rarely found in cosmological models. The result (5.6) is a consequence of the free-falling expanding cosmos. In the next Section we will treat the recession velocities of galaxies and we will return to this ansatz.

If one constructs the Einstein field equations with (5.1), a stress-energy tensor arises on the right side of the form

$$
T_{m^{\prime} n^{\prime}}=-p g_{m^{\prime} n^{\prime}}+\left(p+\mu_{0}\right)^{\prime} u_{m^{\prime}}{ }^{\prime} u_{n^{\prime}} \text {. }
$$

Therein, the pressure and the energy density are 


$$
\kappa p=-\frac{1}{\mathrm{R}^{2}}, \quad \kappa \mu_{0}=\frac{3}{\mathrm{R}^{2}}
$$

from which the equation of state for the cosmic fluid is obtained

$$
p=-\frac{1}{3} \mu_{0}
$$

One gets from the conservation law $T^{m^{\prime} n^{\prime}}=0$ the two subequations

$$
p_{\mid \alpha^{\prime}}=0, \quad \mu_{0 \mid 4^{\prime}}=-3\left(p+\mu_{0}\right) \mathfrak{F}_{4^{\prime}}, \quad \alpha^{\prime}=1^{\prime}, 2^{\prime}, 3^{\prime}
$$

It can be seen that the pressure is position-independent. If the second relation of (5.10) is used for $\mu_{0}$ in (5.10) the ansatz (5.3) is confirmed.

In this Section we have established the field equations for observers who commove with the expansion of the cosmos, and we have also solved this equation and thereby obtained quite simple results. We have already made statements about the curvature of space. However, for their affirmation it is also necessary to treat the problem for non-comoving observers and to make statements about the relative motion of the two observer systems. Only then can we say how the model behaves in free fall and what curvature of space can be read from the metric.

\section{The Non-Comoving System}

The theory of expanding cosmological models is carried out almost exclusively in comoving coordinates. The question arises whether a representation in the non-comoving system is possible. Such a system is usually referred to as static. It was Florides [89], who succeeded in bringing six FRW models into the static form. Mitra [90] [91] has revised the procedure and we [92] [93] have accomplished the coordinate transformations with Lorentz transformations. In the latter are included the physically relevant values for the relative velocities between the two observer systems, i.e. the comoving one and the non-comoving one. Only with them one can make correct statements about the motions in the cosmos and also clarify the question whether superluminal velocities are possible.

We have put up the subluminal model on the static version of the de Sitter model, have passed to the comoving system by a Lemaittre transformation and have abandoned the condition $R=$ const. . If we transform the field equations of this system back into the static form, we cannot assume that we recover the $\mathrm{dS}$ universe. We recall that the subluminal model contains the condition $R=R(t)$. Thus, we will only get the $d S$ model, if we subsequently put $R=$ const. . We will work out this in detail.

In order to present the model in a static system, it is not sufficient, or even necessary, to refer to a static coordinate system. A reference system in rest is required, i.e. a system of four orthogonal vectors in which the quantities of the model can be represented.

The quantities of the comoving system transform with a Lorentz transformation into the non-comoving system. Since the expansion-related relative motion of the galaxies takes place in the radial direction, i.e. in the 1-direction, only the radial and timelike components are occupied in the matrix of the Lorentz trans- 
formation

$$
L_{m}^{m^{\prime}}=\left(\begin{array}{cccc}
\alpha & & & -i \alpha v \\
& 1 & & \\
& & 1 & \\
i \alpha v & & & \alpha
\end{array}\right) .
$$

Therein $\alpha$ is the Lorentz factor and $v$ is the relative velocity of the observers. The relative velocity and the Lorentz factor are taken from the de Sitter model on which our subluminal model is based

$$
v=\frac{r}{\mathrm{R}}, \quad \alpha=\frac{1}{\sqrt{1-r^{2} / \mathrm{R}^{2}}}, \quad a=\frac{1}{\alpha} .
$$

Alternatively, the Lorentz transformation can be derived via the Lemaitre coordinate transformation. The matrix of this coordinate transformation is $\Lambda_{i}^{i^{\prime}}=x^{i^{\prime}}{ }_{\mid i}$, where the $i, i^{\prime}$ are coordinate indices relating to the non-comoving and to the comoving system. From this we obtain with

$$
L_{m}^{m^{\prime}}={\stackrel{m}{e^{\prime}}}_{i^{\prime}}^{\Lambda_{i}}{\stackrel{i}{i^{\prime}}}_{m}^{i}
$$

the matrix of the Lorentz transformation, the 4-beine (tetrads) being read from the metrics (A) and (B), respectively. We have extensively discussed these procedures with the models of the dS family [92] [93].

Therefore, we limit ourselves directly to operations in the local tetrad spaces in order to remain as close as possible to the physically relevant variables, in addition, in expectation that we will not be successful with the coordinate method.

The Ricci-rotation coefficients transform in homogeneously from the comoving to the non-comoving system

$$
A_{m n}{ }^{s}=L_{m n s^{\prime}}^{m^{\prime} n^{\prime} s} A_{m^{\prime} n^{\prime}}^{s^{\prime}}+L_{m n}{ }^{s}, \quad L_{m n}{ }^{s}=L_{s^{\prime}}^{s} L_{n \mid m}^{s^{\prime}} .
$$

The second term in this relation is the Lorentz term. Since the Lorentz transformation is a pseudo-rotation in the [1,4]-subspace, the above relation can be simplified to

$$
U_{m n}{ }^{s}={ }^{\prime} U_{m n}{ }^{s}+L_{m n}{ }^{s} .
$$

The 3-rank quantities $U$ and $L$ can be reduced to 1-rank ones. With

$$
\begin{gathered}
U_{m n}{ }^{s}=h_{m}{ }^{s} U_{n}-h_{m n} U^{s}, \\
L_{m n}{ }^{s}=h_{m}{ }^{s} L_{n}-h_{m n} L^{s}, \quad L_{n}=L_{s n}{ }^{s}=\left\{L_{41}{ }^{4}, L_{14}{ }^{1}\right\}
\end{gathered}
$$

one gains the simple relation

$$
U_{m}={ }^{\prime} U_{m}+L_{m} .
$$

Considering (6.4) and (6.1), one first has

$$
L_{1}=-i \alpha^{2} v_{14}, \quad L_{4}=i \alpha^{2} v_{\mid 1} .
$$

Defining the relative velocity with (6.2) we obtain the auxiliary relations

$$
v_{\mid m}=\{1,0,0,0\} \frac{a}{R}-v \widetilde{F}_{m}, \quad \widetilde{F}_{m}=L_{m}^{m^{\prime}} \widetilde{F}_{m^{\prime}}=\left\{-\alpha v \frac{1}{R}, 0,0,-\alpha \frac{i}{R}\right\}
$$

and finally 


$$
L_{m}=\left\{\alpha^{3} v \frac{1}{\mathrm{R}}, 0,0, i \alpha^{3} \frac{1}{\mathrm{R}}\right\}=\{-i \alpha v, 0,0, \alpha\} i \alpha^{2} \frac{1}{\mathrm{R}}
$$

From (6.7) one obtains

$$
U_{m}=\left\{\alpha^{3} v^{3} \frac{1}{\mathrm{R}}, 0,0, i \alpha^{3} v^{2} \frac{1}{\mathrm{R}}\right\},
$$

a quantity which clearly differs from the static dS expression. However, it can be decomposed according to

$$
U_{m}=\hat{U}_{m}+f_{m}, \quad \hat{U}_{m}=\left\{-\alpha v \frac{1}{R}, 0,0,0\right\}, \quad f_{m}=\left\{i \alpha^{2} v \widetilde{F}_{4}, 0,0,-i \alpha^{2} v \widetilde{F}_{1}\right\}
$$

so that only the $\mathrm{dS}$-expression for the radial force remains after the expansion has been switched off $(\tilde{F}=0)$. At this point it can be discussed whether the radial field quantity in the non-comoving system can be derived from a metric coefficient. If this is not the case, there will be no non-comoving coordinate system. It is easy to find $f_{m}=(\ln \alpha)_{\mid m}$. However, the dS piece $\hat{U}$ of the quantity $U$ is only a gradient if $R=$ const. , i.e. if the subluminal model is reduced to the dS model. We recognize that a Lorentz transformation of the reference system is not always accompanied by a transformation of the coordinate system.

We obtain the lateral field quantities $B$ and $C$ directly from the dS ansatz. Lastly, the subluminal geometry is a snapshot of the dS geometry at any stage of expansion. From (6.4) we obtain, with the usual techniques of the tetrad method,

$$
B_{m}=\left\{\frac{a}{r}, 0,0,0\right\}, \quad C_{m}=\left\{\frac{a}{r}, \frac{1}{r} \cot \vartheta, 0,0\right\}, \quad a=\cos \eta=\sqrt{1-r^{2} / R^{2}}
$$

Alternatively, we can determine these quantities from those of the comoving system with the Lorentz transformation (6.1).

Differentiating (6.10) one obtains the relation

$$
U^{s}{ }_{1}^{s}+U^{s} U_{s}=0
$$

the Friedman equation of the model. A comparison with (5.5) shows that the U-equations are form-invariant under a Lorentz transformation. For the treatment of the B- and C-equations one has to consider again (6.8)

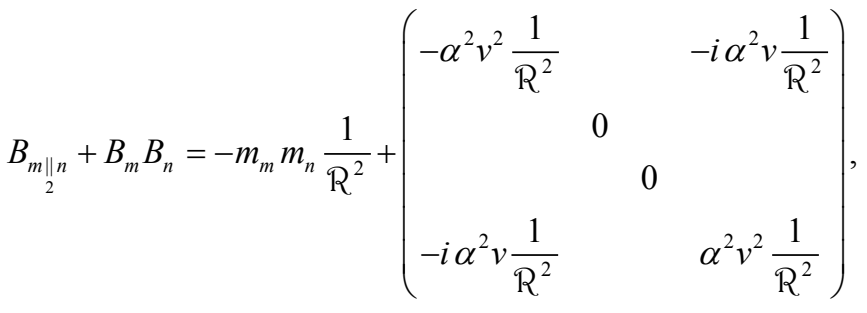

$$
\begin{aligned}
& C_{m \| n}+C_{m} C_{n}=-\left(m_{m} m_{n}+b_{m} b_{n}\right) \frac{1}{\mathrm{R}^{2}}+\left(\begin{array}{lll}
-\alpha^{2} v^{2} \frac{1}{\mathrm{R}^{2}} & & -i \alpha^{2} v \frac{1}{\mathrm{R}^{2}} \\
& 0 & \\
-i \alpha^{2} v \frac{1}{\mathrm{R}^{2}} & & \alpha^{2} v^{2} \frac{1}{\mathrm{R}^{2}}
\end{array}\right) \\
& B_{\substack{\| s \\
s}}+B^{s} B_{s}=-\frac{1}{\mathrm{R}^{2}}, \quad C_{3}^{s}{ }_{3 s}+C^{s} C_{s}=-\frac{2}{\mathrm{R}^{2}} .
\end{aligned}
$$


The relations (5.1) can be used for the field equations. For the stress-energy tensor we expect

$$
\begin{aligned}
& T_{11}=-p-\alpha^{2} v^{2}\left(p+\mu_{0}\right), \quad T_{22}=-p, \quad T_{33}=-p, \\
& T_{41}=-i \alpha^{2} v\left(p+\mu_{0}\right), \quad T_{44}=\mu_{0}+\alpha^{2} v^{2}\left(p+\mu_{0}\right) .
\end{aligned}
$$

With the values from (5.7) the relations (6.12) and (6.13) yield the above expressions.

\section{Discussion of the Model}

In Section 3, we have shown that a metric of the type $k=0$ does not necessarily describe a flat model, but a spatially curved, closed model that expands in free fall. According to the principle of Einstein's elevator observers cannot experience gravitational forces in a free-falling system nor perceive the space as flat.

With our previous results we want to show that these considerations are appropriate for the subluminal model. We put the focus on Section 6, and we take the opposite approach. We transform the field quantities of the non-comoving system into those of the comoving system. We proceed in a step-by-step manner and show that quantities which actually have the properties of curvature can be brought into an apparently flat form.

We use the property of the lateral field quantities to transform homogeneously into the comoving system

$$
B_{m^{\prime}}=L_{m^{\prime}}^{m} B_{m}, \quad C_{m^{\prime}}=L_{m^{\prime}}^{m} C_{m}
$$

and we get, with the Lorentz transformation (6.1), from (6.11)

$$
\begin{gathered}
B_{m^{\prime}}=\left\{\alpha a \frac{1}{r}, 0,0,-i \alpha v a \frac{1}{r}\right\}=\left\{\frac{1}{r}, 0,0,-\frac{i}{R}\right\} \\
C_{m^{\prime}}=\left\{\alpha a \frac{1}{r}, \frac{1}{r} \cot \vartheta, 0,-i \alpha v a \frac{1}{r}\right\}=\left\{\frac{1}{r}, \frac{1}{r} \cot \vartheta, 0,-\frac{i}{\mathrm{R}}\right\}
\end{gathered}
$$

whereby the expressions known from (4.16) are produced in the second step of the calculation. These expressions correspond to a flat geometry which is parameterized with spherical coordinates. Here we have used the relation

$$
\alpha a=1 \text {. }
$$

In this, $\alpha$ is the Lorentz factor, thus a kinematic quantity. On the other hand, $a$ is a geometric quantity which is related to the curvature of space. However, since the relative velocity is linked to the structure of space, the quantities $\alpha$ and $a$ are also linked via (7.3) and simplify the components of the lateral field quantities. Those, however, still contain information about the curvature of space which is hidden by the Einstein elevator effect.

However, the radial field quantities transform inhomogeneously. From the transformation law of the Ricci-rotation coefficients, we obtain the elevator equation

$$
\text { ' } U_{m^{\prime}}=L_{m^{\prime}}^{m} U_{m}+L_{m^{\prime}} .
$$

Its recalculation is quite simple. From (6.10) one obtains with the Lorentz transformation 


$$
U_{m^{\prime}}=L_{m^{\prime}}^{m} U_{m}=\{0,0,0,1\} i \alpha^{2} v^{2} \frac{1}{\mathrm{R}}
$$

and with

$$
L_{m^{\prime}}=-L_{m^{\prime}}^{m} L_{m}=\{0,0,0,1\}\left(-i \alpha^{2} \frac{1}{R}\right)
$$

finally

$$
\text { ' } U_{m^{\prime}}=\left\{0,0,0,-\frac{i}{\mathrm{R}}\right\}
$$

a relation which we have deduced with (5.4) directly from the geometry and which misleadingly suggests a flat space.

It is clear that the use of Einstein's elevator principle is significant in cosmology. It decides whether we live in an infinite cosmos with infinite many stars, or in a finite cosmos with a limited number of stars.

In the following, we hark back to the relations found in (5.6). From $R^{*}=1$, $R *=0$ we have concluded that the expansion of the cosmos is constant. It is now to be investigated how the structure of the cosmos influences the recession velocities of the galaxies, furthermore the significance of this influence for the cosmic horizon, and whether superluminal velocities can occur in the universe.

We start from the relation $r=R \sin \eta$ with the polar angle $\eta$ of the pseudo-sphere which is the basis of the model. If an observer does not perform an individual motion then one has $\eta=$ const. . Differentiation of $r=\mathbb{R} \sin \eta$ leads to the Hubble law with the Hubble parameter $H$

$$
r^{\cdot}=\frac{1}{\mathrm{R}} \mathrm{R}^{\cdot} r=H r
$$

On the other hand, one has, with $R^{\cdot}=1$, according to (6.2)

$$
r^{\cdot}=\frac{r}{\mathrm{R}}=\sin \eta=v
$$

where $v$ has been introduced as a relative velocity in the preceding Sections.

At the equator $(r=\mathbb{R})$ of the pseudo-sphere one has $r^{*}=\mathbb{R}^{*}=1$ or in physical units

$$
v_{H}=r_{H}^{\cdot}=c .
$$

The expansion-induced recession velocity of the galaxies has the highest attainable value, the speed of light, at the equator. A galactic island formation in this model is not possible. The model has a horizon

$$
r_{H}=c T^{\prime} \text {. }
$$

No signal beyond the horizon can reach an observer at $\eta=0$. Since all points on the hyper surface are equivalent, each observer at any position of the universe has his individual horizon.

We also want to survey whether the definition of the velocity

$$
v=r^{\cdot}=\frac{\mathrm{d} r}{\mathrm{~d} T^{\prime}}
$$


(with the proper time $T^{\prime}$ of the comoving observer) conforms to the velocity definition of the theory of relativity. An observer in the non-comoving system detects the radial velocity of a receding galaxy as

$$
v=\frac{\mathrm{d} x^{1}}{\mathrm{~d} T} .
$$

Therein $T$ is its proper time. At each time of expansion, the radial arc element is determined by the $\mathrm{dS}$ metric

$$
\mathrm{d} x^{1}=\alpha \mathrm{d} r, \quad \alpha=1 / a=1 / \cos \eta=1 / \sqrt{1-r^{2} / R^{2}}
$$

The proper times of the observers are linked by the Lorentz relations

$$
\frac{\mathrm{d} T}{\mathrm{~d} T^{\prime}}=\alpha .
$$

The universe expands in free fall, the Lorentz factor $\alpha$ and the metric factor $\alpha$ are identical according to (7.3) and (7.12) so that

$$
v=\frac{\alpha \mathrm{d} r}{\alpha \mathrm{d} T^{\prime}}=r
$$

has been proven as the relative velocity of the observers and thus as the recession velocity of the galaxies.

Remarkably, these results are identical to those derived by Melia [2]-[19] from a model he calls $R_{h}=c t$ model $^{2}$. However, Melia gains his relations from a flat FRW ansatz. In contrast, our subluminal model is positively curved and closed. This has the advantage that Olbers' paradox is neither concealed nor discussed away by expansion effects. The question remains whether both models are identical. We start with our considerations from the dS model, which is based on a pseudo-hyper sphere, i.e., positively curved and closed. According to the FRW classification, however, the expanding version of the dS model is referred to as flat $(k=0)$. In earlier papers [92] [93] we have found that the principle of Einstein's elevator plays an important role in cosmology, and we have used it repeatedly in the preceding Sections. In the light of this method the contradiction between the model of Melia and our subluminal model is resolved. $k=0$ means that the model is based on a positively curved space which expands in free fall and that no gravitational forces are experienced in the comoving system. We can therefore assume that, despite some formal differences, the model of Melia and our subluminal model are identical.

\section{Conclusion}

We have shown that an exact solution to Einstein's field equations exists, a solution which describes an expanding cosmological model which respects the fundamental laws of special and general relativity. In this model, the recession velocity of galaxies cannot exceed the velocity of light. Since the universe is expanding in free fall, no acceleration of the expansion occurs.

${ }^{2}$ Melia's expression agrees with (7.9). Melia's coordinate time $t$ corresponds in the free-falling, comoving system to the proper time of this system. This time is designated by us with $T^{\prime}$. 


\section{References}

[1] Burghardt, R. (2016) Austrian Reports on Gravitation. http://arg.or.at/Wpdf/WTrans3.pdf

[2] Melia, F. and Shevchuk, A.S.H. (2012) Monthly Notices of the Royal Astronomical Society, 419, 2579-2598. https://doi.org/10.1111/j.1365-2966.2011.19906.x

[3] Melia, F. (2012) The $\mathrm{R}_{\mathrm{h}}=\mathrm{ct}$ Universe without Inflation. 1-6. $\underline{\operatorname{arXiv}: 1206.6527 \mathrm{v} 2}$

[4] Melia, F. (2012) Monthly Notices of the Royal Astronomical Society, 422, 14181424. https://doi.org/10.1111/j.1365-2966.2012.20714.x

[5] Melia, F. (2014) The Cosmic Equation of State. arXiv:1411.5771

[6] Melia, F. (2012) The Gravitational Horizon for a Universe with Phantom Energy. arXiv:1206.6192

[7] Melia, F. and Maier, R.S. (2013) Monthly Notices of the Royal Astronomical Society, 432, 2669. https://doi.org/10.1093/mnras/stt596

[8] Melia, F. (2009) International Journal of Modern Physics D, 18, 1113-1127. https://doi.org/10.1142/S0218271809014984

[9] Melia, F. (2016) A Physical Basis for the Symmetries in the Friedmann-Robertson-Walker Metric. arXiv:1601.04991v2

[10] Melia, F. (2012) Proper Size of the Visible Universe in FRW Metrics with Constant Spacetime Curvature. 1-15. arXiv:1207.1332v2

[11] Melia, F. (2012). The Astronomical Journal, 144, 110-117. https://doi.org/10.1088/0004-6256/144/4/110

[12] Melia, F. (2016) The Zero Active Mass Condition in Friedmann-Robertson-Walker Cosmologies. arXiv:1602.01435

[13] Melia, F. (2014) On Recent Claims Concerning the $\mathrm{R}_{\mathrm{h}}=\mathrm{ct}$ Universe. arXiv: $1406.4918 \mathrm{v} 2$

[14] Melia, F. (2016) Cosmological Perturbations without Inflation. arXiv:1606.09565

[15] Melia, F. (2016) Monthly Notices of the Royal Astronomical Society, 463, L61-L63. https://doi.org/10.1093/mnrasl/slw157

[16] Melia, F. (2016) Monthly Notices of the Royal Astronomical Society, 464, 19661976. https://doi.org/10.1093/mnras/stw2493

[17] Melia, F. (2007) Monthly Notices of the Royal Astronomical Society, 382, 19171921. arXiv:0711.4181 https://doi.org/10.1111/j.1365-2966.2007.12499.x

[18] Wei, J.-J., Wu, X.-F., Melia, F. and Maier, R.S. (2015) A Comparative Analysis of the Supernova Legacy Survey Sample with $\Lambda C D M$ and the $\mathrm{R}_{\mathrm{h}}=\mathrm{ct}$ Universe. arXiv:1501.02838

[19] Bikwa, O., Melia, F. and Shevchuk, A. (2012) Monthly Notices of the Royal Astronomical Society, 421, 3356-3361. https://doi.org/10.1111/j.1365-2966.2012.20560.x

[20] Yu, H. and Wang, F.Y. (2014) The European Physical Journal C, 74, 3090-3096. https://doi.org/10.1140/epjc/s10052-014-3090-1

[21] Bilicki, M. and Seikel, M. (2012) Monthly Notices of the Royal Astronomical Society, 425, 1664-1668. https://doi.org/10.1111/j.1365-2966.2012.21575.x

[22] Lewis, G.F. (2013) Monthly Notices of the Royal Astronomical Society, 432, 23242330. https://doi.org/10.1093/mnras/stt592

[23] Lewis, G.F. (2013) Phantom Energy and the Cosmic Horizon: $R_{h}$ Is Still Not a Horizon. $\underline{\text { arXiv:1301.0305 }}$ 
[24] Lewis, G.F. and van Oirschot, P. (2012) Monthly Notices of the Royal Astronomical Society, 423, L26. https://doi.org/10.1111/j.1745-3933.2012.01249.x

[25] Kim, D.O., Lasenby, A.N. and Hobson, M.P. Friedmann-Robertson-Walker Models do not Require Zero Active Mass. arXiv:1601.07890v2

[26] Van Oirschot, P., Kwan, J. and Lewis, G.F. (2010) Monthly Notices of the Royal Astronomical Society, 404, 1633-2176.

[27] Mitra, A. (2014) Monthly Notices of the Royal Astronomical Society, 442, 382-387. https://doi.org/10.1093/mnras/stu859

[28] Weinberg, S. (2008) Cosmology. Oxford University Press, Oxford.

[29] Davis, T.M. (2013) Fundamental Aspects of the Expansion of the Universe and Cosmic Horizons. Thesis.

[30] Davis, T.M. and Lineweaver, C.H. (2001) Superluminal Recession Velocities. arXiv:astro-ph/0011070v2

[31] Davis, T.M., Lineweaver, C.H. and Webb, J.K. (2001) Solutions to the Tethered Galaxy Problem in Expanding Universe and the Observation of Receding Blueshifted Objects. arXiv:astro-ph/0104349

[32] Anderson, J.L. (1996) American Journal of Physics, 64, 527-528. https://doi.org/10.1119/1.18145

[33] Blau, S. (1995) American Journal of Physics, 63, 779-780. https://doi.org/10.1119/1.17822

[34] Callender, C. and Weingard, R. (1995) American Journal of Physics, 63, 780-780. https://doi.org/10.1119/1.17799

[35] Carrera, M. and Giulini, D. (2006) On the Influence of the Global Cosmological Expansion on the Local Dynamics in the Solar System. arXiv:gr-qc/0602098v2

[36] Cooperstock, F.I., Faraoni, V. and Vollick, D.N. (1998) The Astrophysical Journal, 503, 61-66. http://iopscience.iop.org/article/10.1086/305956/pdf https://doi.org/10.1086/305956

[37] Dicke, R.H. and Peebles P.J.E. (1964) Physical Review Letters, 12, 435-437. https://doi.org/10.1103/PhysRevLett.12.435

[38] Irvine, W.M. (1976) Annals of Physics, 33, 322-347.

[39] Mizony, M. and Lachièze-Rey, M. (2005) Astronomy and Astrophysics, 434, 45-52. https://doi.org/10.1051/0004-6361:20042195

[40] Sereno, M. and Jetzer, P. (2007) Physical Review D, 75, Article ID: 064031. https://doi.org/10.1103/PhysRevD.75.064031

[41] Chodorowski, M.J. (2005) American Journal of Physics, 73, 639-643. https://doi.org/10.1119/1.1858487

[42] Cook, R.J. and Burns, M.S. (2009) American Journal of Physics, 77, 59-66. https://doi.org/10.1119/1.2987790

[43] Endean, G. (1995) Monthly Notices of the Royal Astronomical Society, 277, 627-629.

[44] Harrison, E. (1993) Astrophysical Journal, 403, 28-32. https://doi.org/10.1086/172179

[45] Kiang, T. (2003) Can we Observe Galaxies That Recede Faster than Light? - A More Clever-Cut Answer. arXiv:astro-ph/0305518v1

[46] Lewis, G.F., et al. (2006) Coordinate Confusion in Conformal Cosmology. arXiv:0707.2106v1

[47] Murdoch H.S. (1977) Quarterly Journal of the Royal Astronomical Society, 18, 242 - 
247.

[48] Silverman, A.N. (1986) American Journal of Physics, 54, 1092-1096. https://doi.org/10.1119/1.14721

[49] Stuckey, W.M. (1992) American Journal of Physics, 60, 142-146. https://doi.org/10.1119/1.16933

[50] Liebscher, D.-E. (2007) Astronomische Nachrichten, 328, 586-587. https://doi.org/10.1002/asna.200710752

[51] Rindler, W. (1956) Monthly Notices of the Royal Astronomical Society, 116, 662677. https://doi.org/10.1093/mnras/116.6.662

[52] Barnes, L.A., Francis M.J., James, J.B. and Lewis G.F. (2006) Monthly Notices of the Royal Astronomical Society, 373, 382-390. https://doi.org/10.1111/j.1365-2966.2006.11045.x

[53] Ellis, G.F.R. and Rothman, T. (1993) American Journal of Physics, 61, 883-893. https://doi.org/10.1119/1.17400

[54] Ellis, G.F.R. and Stoeger, W. (1988) Classical and Quantum Gravity, 5, 207-220. https://doi.org/10.1088/0264-9381/5/1/024

[55] Harrison, E. (1991) Astrophysical Journal, 383, 60-65. https://doi.org/10.1086/170763

[56] Shi, X. and Turner, M.S. (1998) Astrophysical Journal, 493, 519-522. https://doi.org/10.1086/305169

[57] Whiting, A.B. (2004) The Expansion of Space: Free Particle Motion and the Cosmological Redshift. arXiv:astro-ph/0404095

[58] Harvey, A., Schucking, E. and Surowitz, E.J. (2005) Redshift and Killing Vectors. arXiv:gr-qc/0508125

[59] Bunn, F.B. and Hogg, D.W. (2009) American Journal of Physics, 77, 688-694. https://doi.org/10.1119/1.3129103

[60] Chodorowski, M.J. (2006) A direct Consequence of the Expansion of Space? arXiv:astro-ph/0610590v3

[61] Chodorowski, M.J. (2011) Monthly Notices of the Royal Astronomical Society, 413, 585-594. https://doi.org/10.1111/j.1365-2966.2010.18154.x

[62] Faraoni, V. (2010) General Relativity and Gravitation, 42, 851-860. https://doi.org/10.1007/s10714-009-0885-8

[63] Narlikar, J.V. (1994) American Journal of Physics, 62, 903-907. https://doi.org/10.1119/1.17679

[64] Bondi, H. (1999) Monthly Notices of the Royal Astronomical Society, 302, 337-340. https://doi.org/10.1046/j.1365-8711.1999.02137.x

[65] Endean, G. (1994) Astrophysical Journal, 434, 397-401. https://doi.org/10.1086/174741

[66] Infeld, I. and Schild, A. (1945) Physical Review, 68, 250-272. https://doi.org/10.1103/PhysRev.68.250

[67] Querella, L. (1998) Astrophysical Journal, 508, 129-131. https://doi.org/10.1086/306376

[68] Abramowicz, M.A., Bajtlik, S., Lasota, J.-P. and Moudens, A. (2006) Eppur si espande. arXiv:astro-ph/0612155v3

[69] Abramowicz, M.A., Bajtlik, S., Lasota, J.-P. and Moudens, A. (2008) Eppur si espande. arXiv:0812.3266v1

[70] Aspden, H. (1988) American Journal of Physics, 56, 584. 
https://doi.org/10.1119/1.15520

[71] Chodorowski, M.J. (2006) Is Space really expanding? A Counterexample. arXiv:astro-ph/0601171v2

[72] Chodorowski, M.J. (2006) Eppur si muove. arXiv:0812.3972v1

[73] Epstein, L.E. (1987) American Journal of Physics, 55, 970. https://doi.org/10.1119/1.14900

[74] Felten, J.E. and Isaacman, R. (1986) Reviews of Modern Physics, 58, 689-689. https://doi.org/10.1103/RevModPhys.58.689

[75] Harvey, A. (1988) American Journal of Physics, 56, 487-488. https://doi.org/10.1119/1.15589

[76] Peacock, J.A. (2008) A Diatribe on Expanding Universe. arXiv:0809.4573v1

[77] Stuckey, W.M. (1992) American Journal of Physics, 60, 554-560. https://doi.org/10.1119/1.17123

[78] Harrison, E.R. (1964) Nature, 204, 271-272. https://doi.org/10.1038/204271b0

[79] Wesson, P.S., Valle, K. and Strabell, R. (1987) Astrophysical Journal, 317, 601-606. https://doi.org/10.1086/165306

[80] Wesson, P.S. (1986) Space Science Reviews, 44, 169-176. https://doi.org/10.1007/BF00227231

[81] Burghardt, R. (2016) Austrian Reports on Gravitation. http://arg.or.at/Wpdf/Wcurv.pdf

[82] Burghardt, R. (2016) Journal of Modern Physics, 7, 2347-2356. https://doi.org/10.4236/jmp.2016.716203

[83] Liebscher, D.-E. and Treder, H.-J. (1970) General Relativity and Gravitation, 1, 117 125. https://doi.org/10.1007/BF00756890

[84] Treder H.-J. (1966) Lorentz Gruppe, Einstein Gruppe und Raumstruktur. DAW Einsteinsymposium, 57-75.

[85] Burghardt, R. (2005) Spacetime Curvature. http://arg.or.at/EMono.htm, http://arg.or.at/Mono.htm

[86] Kopeikin, S.M. (2013) Einstein's Equivalence Principle in Cosmology. arXiv:1311.4912v3

[87] Kopeikin, S.M. (2012) Celestial Ephemerides in an Expanding Universe. arXiv: 1207.3873

[88] Kopeikin, S.M. (2013) Equivalence Principle in Cosmology. arXiv:1307.5360

[89] Florides, P.S. (1980) General Relativity and Gravitation, 12, 563-574. https://doi.org/10.1007/BF00756530

[90] Mitra, A. (2015) International Journal of Modern Physics D, 24, Article ID: 1550032. https://doi.org/10.1142/S0218271815500327

[91] Mitra, A. (2013) Gravitation and Cosmology, 19, 134-137. https://doi.org/10.1134/S0202289313020072

[92] Burghardt, R. (2016) Austrian Reports on Gravitation. http://arg.or.at/Wpdf/WTrans1.pdf

[93] Burghardt, R. (2016) Austrian Reports on Gravitation. http://arg.or.at/Wpdf/WTrans2.pdf 